\title{
Review on Self Emulsifying Drug Delivery System for Delivery of Drugs
}

\author{
Rahul Shukla ${ }^{1 *}$, Chukwunedu Chioma Faith ${ }^{1}$, Richa Srivastava ${ }^{1}$ and Shweta ${ }^{2}$ \\ ${ }^{1}$ Amity Institute of Pharmacy, Amity University Uttar Pradesh, India \\ ${ }^{2}$ Sri Venkateswara University, India \\ *Corresponding author: Rahul Shukla, Amity Institute of Pharmacy, Amity University Uttar Pradesh Lucknow Campus, India
}

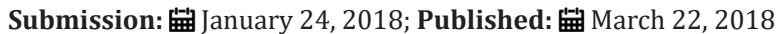

\begin{abstract}
The oral route of administration of drugs is the most common and easiestroute of administration, because of this reason most drugs are administered via oral route. Drugs administered orally should possess very good aqueous solubility for better absorption and bioavailability. But studies have shown that up to $35 \%-40 \%$ of new drugs possess poor aqueous solubility which leads to poor bioavailability. Self-emulsifying drug delivery systems are novel drug delivery systems that were created with the purpose of enhancing the bioavailability of poor aqueous soluble drugs. A lot of attention and focus has been put into this delivery system lately. SEDDS belong to the group of lipid-based formulations.

The unique feature of this delivery system is the ability to self-emulsify, that is, their ability to form micro emulsions or oil-in-water emulsions when diluted in the aqueous phase because of the gentle agitation of the gastrointestinal tract used for hydrophobic drugs having dissolution rate-limited absorption. SEDDS may have future potential in increasing the rate and extent of absorption of orally administered drugs. Low cost self-emulsifying drug delivery systems have easily accessible excipients such as natural oils or synthetic oils, surfactants and co-surfactants are now being formulated.
\end{abstract}

This article aims to review research works on self emulsifying drug delivery system, its pharmaceutical applications in the delivery of drugs, its merits and demerits, mechanism of action, different dosage forms, factors affecting its manufacturing and process.

Keywords: Self emulsifying drug delivery system (SEDDS); Lipid-based formulations; Bioavailability

\section{Introduction}

The need for increased folds in bioavailability of oral lipophilic drugs led to studies on self-emulsifying drug delivery system. Drugs which have low solubility in aqueous medium but high permeability have given rise to self-emulsifying drug delivery systems. Selfemulsifying drug delivery systems are also known as SEDDS. They are isotropic mixtures of drug, oil, solid or liquid surfactants and hydrophilic solvents or co-solvents. The first marketed SEDDS is cyclosporine and it was found to have higher bioavailability than the conventional drug. Self-emulsifying drug delivery systems in research or development include formulations of drugs [1].

Self-emulsifying drug delivery system can be administered orally via soft or hard gelatin capsules. When they get diluted in aqueous medium, due to the gentle churning of gastrointestinal fluids they form relatively fine oil-in-water emulsions. This is the process of self-emulsification. Emulsions are liquid dosage forms which consist of two immiscible phases; where one is a dispersed phase is dispersed into the other phase, dispersion medium, and stability is maintained with the help of an emulsifying agent. The process of self-emulsification can be better explained with the ouzo effect which occurs in anise-flavored liquors where an oil-in-water emulsion is formed when the anise comes in contact with water.

They are used to improve oral absorption of highly lipophilic drugs which have low aqueous solubility. The future of selfemulsifying drug delivery system in the pharmaceutical market is almost guaranteed because studied show that about $40 \%$ of drug compounds newly produced are hydrophobic in nature. This shows that studies relating to self-emulsifying drug delivery system would continue and more drugs will be formulated as SEDDS [2].

The bioavailability of hydrophobic drugs is enhanced by the presence of fatty acids, e.g. intake of lipid-rich meals with drugs, lipid-based drug delivery systems are formulated form poor water-soluble drugs that are administered orally. Under the lipid formulation classification systems, Type III formulations form the self (micro) emulsifying drug delivery systems which are clear isotropic mixtures of oils, hydrophilic solvents, poor water-soluble drugs and hydrophilic surfactants. These self-emulsifying drug delivery systems help to protect the drug from metabolism such as chemical or enzymatic hydrolysis along the GIT till it reaches the intestine where it is absorbed. It also solubilizes the drugs and increases the bioavailability. SEDDS are easily manufactured and cheap. This is an advantage for developing countries [1] (Table 1).

\section{Merits of SEDDS}

a. The main advantage of SEDDS is that it enhances the bioavailability of oral lipophilic drugs. 
b. Drugs formulated using SEDDS selectively target site by heading towards specific absorption sites in GIT.

c. SEDDS lead to enhanced solubility by making lipophilic drugs more soluble in aqueous medium.

d. Drugs formulated into SEDDS are protected from the hostile environment in the gut. The gut has metabolic function. Sensitive drugs can be formulated into SEDDS for protection.

e. Food affects drug action by causing variability. SEDDS reduce the variability of drug action.

f. SEDDS have the ability to rapidly self-emulsify when

Table 1: Marketed Preparations of SEDDS.

\begin{tabular}{|c|c|c|c|}
\hline Brand Name & Generic Drugs & Dosage Forms & Manufacturer \\
\hline Convulex & Valproic acid & Soft gelatin capsule & Gerot Pharmazeutika \\
\hline Norvir & Ritonavir & Soft gelatin capsule & Abbvie, Abbot \\
\hline Fortovase & Saquinavir & Soft gelatin capsule & Hoffman- Roche \\
\hline Neoral & Cyclosporine & Soft gelatin capsule & GSKartis \\
\hline Agenerase & Amprenavir & Soft gelatin capsule & Sanofi- Aventis \\
\hline Lipirex & Fenofibrate & Hard gelatin capsule & Sanofi- Aventis \\
\hline Solufen & Ibuprofen & Soft gelatin capsule & Abbvie \\
\hline Depakene & Valproic acid & Soft gelatin capsule & Hoffman Le Roche \\
\hline Accutane & Isotretinoin & Soft gelatin capsule & Virtus \\
\hline Prometruim & Progesterone & The sollibity and \\
\hline
\end{tabular}

\section{Disadvantages of SEDDS}

a. The high content of surfactant presents in self-emulsifying drug delivery system which ranges between 30\% - 60\% irritates the GIT.

b. In-vitro models of self-emulsifying formulations lack good predicative studies on assessment of the formulation.

c. Co-solvents which are volatile in nature can migrate on the soft or hard gelatin capsule shell leading to the precipitation of lipophilic drug.

d. The usual dissolution evaluation tests do not work because SEDDS formulations potentially depend on digestion before the release of the drug.

e. Chemical instabilities are observed in the self-emulsifying drug delivery systems.

f. Production cost is expensive.

g. Self-emulsifying drug delivery system formulations containing a high number of components become difficult to validate.

h. Drug incompatibility is low.

i. Leakage of drug may occur which leads to lesser drug loading [4].

\section{Factors affecting the efficiency of oral absorption}

These factors also determine the drug's ability to self-emulsify. in contact with gastro intestinal fluids and due to the influence of gentle churning provided by peristaltic and other gastrointestinal movements, a fine oil-in-water emulsion is formed.

g. Hydrophilic or hydrophobic drugs can be effectively incorporated within the oil-surfactant mixture.

h. SEDDS can be formulated into liquid dosage forms and also solid dosage forms.

i. Compared to the conventional dosage forms, drugs formulated as SEDDS are administered in lower doses [3].
I. The solubility and dose of the drug- Unless a drug exhibits extremely good solubility in any one or more components, which make up the SEDDS formulations, most importantly the oil phase, drugs that are administered in high dose are not suitable for SEDDS. Drugs that have low solubility in water and oils and exhibit limited solubility with $\log$ P values having an estimate of 2 are more difficult to deliver by SEDDS. The solubility of the drug in the oil phase determines the ability of SEDDS to keep the drug in solubilized form [2].

II. The concentration of surfactant or co-surfactantPrecipitation may occur if the surfactant or co-surfactant contributes greatly to the solubilization of drug as this leads to low capacity of surfactant or co-surfactant to act as solvent due to the dilution of SEDDS.

III. The polarity of emulsion- The polarity of the emulsion depends on the polarity of the lipophilic phase and this controls the release of drugs from the emulsions.

IV. The size, polarity and charge of the droplet- Factors that control the polarity of the droplet include hydrophilic-lipophilic balance, molecular weight of the micronized drug, and the degree of unsaturation and chain length of fatty acid.

V. The temperature at which self-emulsification occurs [5].

\section{Process of Self Emulsification}

\section{Self nano emulsifying drug delivery system (SNEDDS)}

These are Nano emulsions formed from SEDDS. Self-Nano 
emulsifying drug delivery systems are heterogeneous dispersions of two immiscible liquids which have a mean droplet size that falls within the Nano metric scale $(20-200 \mathrm{~nm})$. Self-emulsifying drug delivery systems are important for increasing the solubility of drugs [6].

\section{Self micro emulsifying drug delivery system (SMEDDS)}

They form micro emulsions when in contact with water. The emulsions formed from SMEDDS have a mean droplet size that falls within the micrometric scale which ranges between $2-100 \mathrm{~nm}$. The main difference between common emulsions and micro emulsions is the mean droplet size. SMEDDS are thermodynamically stable. They form optically transparent emulsions. Because of the small droplet size, surface area for absorption and dispersion are increased significantly and it easily penetrates the gastrointestinal tract and can be absorbed [1].

\section{Composition of Self Emulsifying Drug Delivery System}

The self-emulsification process is specific to the factors affecting self-emulsification and all factors should be considered in the selection of excipients.
a. Drug or Active Pharmaceutical Ingredient
b. Natural or synthetic oils
c. Solid or liquid surfactants
d. One or more hydrophilic solvents/co-solvents.

\section{Drug/active pharmaceutical ingredient}

According to the Biopharmaceutical classification system (BCS), there are four classes of drugs based on solubility (ability of a solute dissolve in a solvent) and permeability (contact between a solute and solvent to form a solution) [4]. These classes include

a. Class I- High solubility and high permeability

b. Class II- Low solubility and high permeability

c. Class III- High solubility and low permeability

d. Class IV- Low solubility and low permeability

The class II drugs which have low solubility and high permeability are used in the formulation of SEDDS [1]

\section{Oil}

Oil is by far the most important excipient. It is responsible for facilitating the process of self-emulsification. Because of the importance of oil in SEDDS, they are also called self-emulsifying oil formulations. It helps in the solubilizing lipophilic drugs. Natural or synthetic oils can be used in self-emulsifying drug delivery system. They increase the fragments of lipophilic drugs that pass through the intestinal lymphatic system; this increases the absorption from gastrointestinal tract depending on the nature of triglyceride.

Different degrees of saturation of low chain triglyceride (LCT) and Medium chain triglyceride (MCT), monoglycerides, diglycerides have been used in the formulations of SEDDS. Semi synthetic medium chain triglycerides are novel compounds. They are defined as compounds having both hydrophilic and lipophilic properties as well as having surfactant properties. Novel semi-synthetic medium chain triglycerides are amphilic compounds and they are rapidly replacing the regular MCT oils [7].

Oils affect the bioavailability of oral lipophilic drugs by increasing the intestinal lymphatic permeability, solubility in gastric and intestinal fluids, protecting the drug from metabolism and increasing the rate of dissolution. Generally, a higher concentration of surfactants like cremophor RH40 is required when using low chain triglycerides to form micro-emulsions compared to medium chain triglycerides. Hydrolyzed vegetable oils are widely used since they form a good emulsification system with vast number of approved orally administered surfactants. They also show better drug solubility properties [2]

Comestible oils are not usually selected because their ability to dissolve large quantities of lipophilic drugs is poor. Hydrolyzed oils provide more advantages and their degraded products are almost similar to the natural end products of digestion. Some oils that can be used in the composition of SEDDS include
a. Corn oil
b. Sesame oil
c. Soybeans oil
d. Olive oil
e. Peanut oil
f. Hydrogenated vegetable oil
g. Hydrogenated soybeans oil

\section{Surfactants}

In the formulation of SEDDS, non-ionic surfactants having high value in the hydrophilic-lipophilic balance (HLB) scale are used. Non-ionic surfactants are preferred to cationic and anionic surfactants in SEDDS because they are not harmful. The concentration of surfactants added to SEDDS formulation are $30 \%$ $60 \%$. This high concentration of surfactants leads to irritation of the gastrointestinal tract and is a drawback of SEDDS [6].

Safety is a huge criterion in choosing surfactant. The choice is restrained as very few surfactants are approved as orally acceptable. No-ionic surfactants are widely recommended because they are less toxic compared to ionic surfactants but they may alter the permeability of the intestinal lumen by reversible changes. Surfactants of natural sources are usually preferred to synthetically made surfactants but the natural emulsifiers have restrained selfemulsification ability. Studies show a relationship between the mean droplet size and the concentration of surfactants used. In some cases, the increase in the surfactant concentration leads to a decrease in mean droplet size as seen in SMEEDS. This is because of the stability of the oil droplets due to the positioning of surfactant molecule in oil-water interface [1]. In other cases, an increase in surfactant concentration leads to an increase in mean droplet size. 
This can be explained by the disruption on the interface educed by enhanced water penetrations into oil droplets by which the increased surfactant concentration acts as a medium leading to the extrusion of oil droplets into the aqueous phase.

Surfactants employed for use in the SEDDS formulation act by various mechanisms to improve the bioavailability which include increased permeability of intestinal epithelium, reduced or inhibited p-glycoprotein drug efflux, enhanced drug dissolution and increased tight junction permeability. High quantity of surfactant can cause reversible changes in the permeability of intestinal wall. Solid or liquid surfactants maybe used in SEDDS formulation. The non-ionic group of surfactants consists of tween and span. They include
a. Tween 20 (Poly sorbate 20 )
b. $\quad$ Tween 80 (Poly sorbate 80 )
c. Span 80 (Sorbitan mono-oleate)
d. Hydrogenated castor oil
e. D-alpha Tocopheryl
f. Cremophor RH40

\section{Co-solvents}

Co-solvents are solvents that help in dissolving immiscible phases (oil/aqueous) in a formulation. They dissolve either large amounts of hydrophilic surfactants or the hydrophobic drug in oil phase. One or more hydrophilic solvents maybe used. Co-solvents can also be referred as co-surfactants depending on their use in a formulation.

Because high concentration of surfactants is required in SEDDS formulations, usually above $30 \%$, which causes irritation in the gastrointestinal tract, co-surfactants are employed to reduce the concentration of surfactants. Both surfactants and co-surfactants work together to reduce the interfacial tension to a negligible negative value [8].

When this value is achieved, the interface expands to form droplets that are finely dispersed, surfactants and co-surfactants are later adsorbed until the bulk condition is exhausted enough to make a positive interfacial tension. This is called spontaneous emulsification and it forms the emulsions. In self emulsifying drug delivery system, organic solvents that are approved for oral administration such as polyethylene glycol, ethanol and propylene glycol can act as co-surfactants dissolving large quantities of either the drug in oil base or the hydrophilic surfactant.

Studies show that there are alcohol-free self -emulsifying emulsions. These alcohol-free SEDDS systems have advantages over the other formulations because in capsule dosage forms, alcohol and volatile solvents migrate to the soft or hard gelatin capsule shell causing precipitation of lipophilic drug. In alcohol-free formulation systems, lipophilic drug dissolution is limited. Proper choice should be considered in the selection of excipients [3]. Some co-solvents that are used include
a. Ethanol
b. Propylene glycol
c. Poly ethylene glycol (PEG)
d. Glycerin

Other excipients added to SEDDS formulations include;

\section{Viscosity enhancers}

Viscosity enhancers are components that added to selfemulsifying drug delivery systems to alter the viscosity of the formulations. Some examples of viscosity enhancers include;
a. Tragacanth
b. Beeswax
c. Acetyl alcohol
d. Stearic acids

\section{Antioxidants}

In SEDDS formulations, antioxidants of lipophilic nature are added to stabilize the oil phase of the formulations. Examples include;
a. Tocopherol
b. Ascorbic palmitate
c. Propyl gallate

\section{Polymers}

Inert polymers which are non- ionizable at physiological $\mathrm{pH}$ present $5 \%$ to $40 \% \mathrm{w} / \mathrm{w}$ are able to form matrix. When added to SEDDS formulation, it prevents precipitation by formulating super saturable SEDDS [1]. Polymers include;

a. Hydroxyl propyl methyl cellulose

b. Ethyl cellulose (EC)

\section{Development of Solid Self Emulsifying Drug Delivery Systems}

One of the advantages of self-emulsifying drug delivery systems is that it can be formulated as liquid dosage forms as well as solid dosage forms. Usually, all the ingredients used in self-emulsifying drug delivery systems are usually in liquid forms, e.g., oils, liquid surfactants and co-solvents, because of this, most SEDDS are in liquid state which can pose some disadvantages like precipitation, low stability and high costs [6].

Solid self emulsifying drug delivery systems (S-SEDDS) are now manufactured and have gained popularity because they eliminate all the disadvantages that come with the liquid dosage forms of SEDDS. For the preparation of solid emulsifying drug delivery systems, the liquid dosage forms are solidified to create solid dosage forms [7]. The merits of SEDDS and the advantages of solid dosage forms are merged together to form solid self emulsifying drug delivery systems. 


\section{Solidification techniques used in the transformation of liquid SEDDS to solid SEDDS}

Capsule filling with liquid and semi-solid self emulsification formulations: This is the most common and easiest solidification techniques. It involves the filling of liquid or semi-solid self emulsifying formulations into hard or soft capsule shells. For liquid self emulsification formulations, a technology technique is used which is called Liquid- Oros technology which used osmotic properties where the layer expands when it comes in contact with water and is pumped into the hard or soft gelatin capsules.

For the semi-solid self emulsifying formulations, the semisolids are heated to a temperature twenty degrees above their melting point. The molten mixture is placed in the capsule shell with a stirrer. The capsule is capped and left to cool [7].

Spray drying: All the ingredients, that is, excipients, drug and solid carriers are mixed and solubilized. The solubilized mixture is put in a spray dryer and atomization of droplets occurs. The water phase in the emulsion evaporates and the particles formed are dried in the drying chamber. The particles are collected and used for the formulations of self emulsifying tablets and capsules [1].

Adsorption to solid carriers: The adsorption to solid carrier technique is another simple technique for preparation of self emulsifying powders. It requires the mixing of liquid self emulsifying formulations and solid carriers in a blender. The resultant can further be used in the formulation of self emulsifying tablets and capsules. Liquid self emulsifying formulations easily adhere to the solid carriers. The solid carriers that are used include cross-linked polymers, such as cross-linked sodium carboxyl methyl cellulose, cross-linked povidone, or nano-particles absorbents such as charcoal, bamboo charcoal, porous silicon dioxide [2].

Melt granulation: Unlike wet granulation, the binding agent used in melt granulation melts at low temperature and is used to form powder agglomeration. It has more advantages than wet granulation. It is also known as "one-step" technique [6].

Melt-extrusion/extrusion spheronization: This is a solventfree technique. The extrusion-spheronization includes drying of the ingredients, that is, drugs and excipients, addition of a liquid binder to wet the mixture. The mixture is then extrudated with pressure and controlled temperature. The spheronization occurs when the extrudate form spheroids of same size. These spheroids are dried and may be coated. The melt extrusion/extrusion spheronization technique is used in the pharmaceutical industry. Self emulsifying pellets of Diazepam and progesterone were manufactured with the melt-extrusion/extrusion-spheronization technique [7].

\section{Dosage Forms of Self Emulsifying Drug Delivery System}

\section{Self emulsifying capsules}

These are typical liquid self-emulsifying formulations encapsulated in soft or hard gelatin capsule shells. When administered, they spontaneously form fine droplets of micro emulsions. These micro-emulsions are dispersed in the gastrointestinal tract and improve intestinal absorption. Irrefutable limitations in this dosage form which cause decrease in drug absorption is the irreversible phase separation of micro-emulsion which may take place [5].

In cases where this may occur, the anionic surfactant, sodium dodecyl sulphate is added to the self-emulsifying formulations to improve absorption. A small quantity of polymer is used in the formulation to formulate super-saturable SEDDS to prevent precipitation of drug ensuring a supersaturated state is generated and maintained in vivo. These formulations have a decreased amount of surfactant so the side effects relating to the gastrointestinal tract are minimized.

Apart from filling in liquid formulations in capsules, the liquid self emulsifying formulations can also be filled in solid or semisolid state by combining the liquid with a solid carrier. Oral SEDDS capsules were found to have a higher patient compliance than injections as it was seen in Low Molecular Weight Heparin (LMWH) which was usually administered by parenteral route. The LMWH was merged with solid carrier absorbents and placed into hard capsule shells. This technique was also applied to Gentamicin which is usually administered in topical or parenteral route. Gentamicin self emulsifying capsules were formulated [3].

\section{Dry emulsions}

Dry emulsions are oil-in-water emulsions that use techniques like spray drying, rotatory evaporation freeze drying or solid carrier adsorption to get converted into actual powders. They are solid dosage forms. Before use, these powders maybe re-dispersed into water. Dry emulsions are powders that undergo self-emulsification in vivo or when they make contact with aqueous solution.

In rotator evaporation technique, mineral oils and sucrose are used to obtain glass emulsions in the form of dry foams. Surfactants are not required in this technique. Spray-drying technique is mostly used in the formulations of dry emulsions. Currently, dry emulsions were prepared by spreading liquid self emulsifying formulations on a glass plate and left to dry and further mixed to powders [4]. This dry emulsion technology neglects the use of toxic organic solvents and eradicates all the stability problems associated with a typical emulsion like creaming, phase separation, micro-organism contamination during storage. For the oil phase of dry emulsion formulations, medium chain triglycerides (MCT) are used. Dry emulsions can be used to further the formulation of tablets and capsules.

\section{Self emulsifying solid dispersion}

Although stability is the main interest in the manufacturing process, they are used to increase the rate of dissolution and also the bioavailability of drugs that are poorly soluble in water. A technique that is commonly used for the preparation of self-emulsifying solid dispersion is the hot-melt granulation. Self emulsifying solid dispersions may be filled in capsules in the molten form [2].

\section{Self emulsifying sustained-release tablets}

The self-emulsifying tablets have been successfully formulated. The aim was to create self-emulsifying tablets that would not 
require a large amount of solid excipients, and for this, a gelling agent called colloidal silicon dioxide was introduced. The gelling agent helped to minimize the amount of solid excipients required for the formulations of self-emulsifying tablets and also to cause slow or sustained release of drug, hence the name.

Indomethacin is a hydrophobic non-steroidal anti inflammatory drug (NSAID). Application of Indomethacin self emulsifying tablets could increase permeability through gastrointestinal mucosa to avoid bleeding. A new technology is introduced in self emulsifying tablets involving the use of osmotic pump as carriers [3].

\section{Self emulsifying sustained-release pellets}

Pellets have a lot of advantages such as ease of dispersion in gastrointestinal tract, flexible manufacturing environment. Therefore, the need to merge the good characteristics of pellets with the characteristics of SEDDS led to the formulation of selfemulsifying sustained release pellets. Self emulsifying pellets were prepared using the extrusion/spheronization [9].

\section{Self emulsifying nanoparticles}

Self emulsifying nanoparticles have been formulated by a technique which involves the melting of drug, lipid and surfactants together, the mixture is injected in a stirred solvent dropwise. The resulting nanoparticles were filtered and dried. The technique employed in self emulsifying nanoparticles is known as the solvent injection technique. A second technique is called sonication emulsion-diffusion-evaporation.

\section{Self emulsifying suppositories}

Some studies have shown that solid self-emulsifying drug delivery systems which can increase gastrointestinal absorption also have the properties to increase rectal and vaginal absorption [7]. Example; Glycyrrhizin would give a better therapeutic value of chronic hepatic disease when administered as self emulsifying suppositories.

\section{Self emulsifying implants}

Self-emulsifying implants have shown advancement in solid self-emulsifying drug delivery systems. Co-polymers that have a hydrophilic region and about 2 functional groups that can be crosslinked are used in the manufacture of self-emulsifying implants. These co-polymers are used as sealants.
Carmusine, a chemotherapeutic agent has a short half-life and is used for brain tumors [2]. Self emulsifying carmusine or Bis chloroethyl nitorosourea was formulated by compression molding to form wafer-like implants.

\section{Conclusion}

This review paper studies on self-emulsifying drug delivery systems emphasizing on its main advantage; to improve the bioavailability of poor water-soluble drugs. SEDDS also improve the solubility and absorption of the lipophilic drug as well as intestinal permeability. There are several marketed products of SEDDS in the market of which most are in capsule dosage forms. Solid self emulsifying drug delivery systems are preferred to liquid formulations because the disadvantages of liquid SE formulations are eliminated. SEDDS are used for drugs with low solubility and high permeability.

\section{References}

1. Ajay K, Surabh S, Ravindra K (2012) Self-emulsifying drug delivery systems (SEDDS): Future aspects', International Journal of Pharmacy and Pharmaceutical sciences 2(4): 7-13.

2. Pouton CW (2006) Formulation of poorly water soluble drugs for oral administration: physicochemical and physiological issues and the lipid formulation classification system. Eur J Pharm Sci 29(3-4): 278-287.

3. Gursoy RN, Benita S (2004) Self-emulsifying drug delivery systems (SEDDS) for improved oral delivery of lipophilic drugs. Biomed Pharmacother 58(3): 173-182.

4. Kshitija K, Swati M (2013) Self-emulsifying drug delivery system: a review. International Journal of Pharmaceutical sciences and Researc 4(12): 4494-4507.

5. Patel PA, Chaulang GM, Akolkotkar A, Mutha SS, Hardikar SR, et al. (2008) Self-emulsifying drug delivery system: a review. J Pharm and Tech 1(4): 313-323

6. Patrick B, Memvanga, Véronique P (2012) Formulation design and in vivo antimalarial evaluation of lipid-based drug delivery systems for oral delivery of beta-arteether. Eur J Pharm Biopharm 82(1): 112-119.

7. Pouton CW, Porter CJ (2008) Formulation of lipid-based delivery systems for oral administration: materials, methods and strategies. Adv Drug Deliv Rev 60(6): 625-637.

8. Porter CJ, Charman WN (2001) In vitro assessment of oral lipid based formulations. Adv Drug Deliv Rev 50(Suppl 1): S127-S147.

9. Eckstein Ludwig U, Webb RJ, Van Goethem ID, East JM, Lee AG, et al (2003) Artemisinins target the SERCA of Plasmodium falciparum. Nature 424(6951): 957-961.
Creative Commons Attribution 4.0 International License

For possible submissions Click Here

\section{Submit Article}

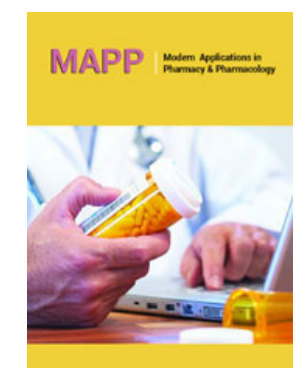

Modern Applications in Pharmacy \& Pharmacology

\section{Benefits of Publishing with us}

- High-level peer review and editorial services

- Freely accessible online immediately upon publication

- Authors retain the copyright to their work

- Licensing it under a Creative Commons license

- Visibility through different online platforms 